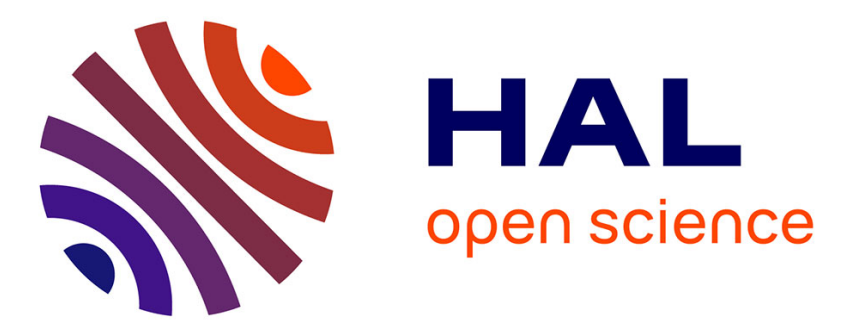

\title{
La fin du partage? Les capitalismes de la copie face au capitalisme de la rente globale: une nouvelle géographie des industries de santé
}

Maurice Cassier

\section{- To cite this version: \\ Maurice Cassier. La fin du partage? Les capitalismes de la copie face au capitalisme de la rente globale: une nouvelle géographie des industries de santé. Mouvements : des idées et des luttes, 2019. halshs-02162781}

\author{
HAL Id: halshs-02162781 \\ https://shs.hal.science/halshs-02162781
}

Submitted on 22 Jun 2019

HAL is a multi-disciplinary open access archive for the deposit and dissemination of scientific research documents, whether they are published or not. The documents may come from teaching and research institutions in France or abroad, or from public or private research centers.
L'archive ouverte pluridisciplinaire $\mathbf{H A L}$, est destinée au dépôt et à la diffusion de documents scientifiques de niveau recherche, publiés ou non, émanant des établissements d'enseignement et de recherche français ou étrangers, des laboratoires publics ou privés. 


\section{La fin du partage ? Les capitalismes de la copie face au capitalisme de la rente globale : une nouvelle géographie des industries de santé}

\section{Maurice Cassier}

La géographie des industries de santé a été bouleversée depuis les années 1970 par l'émergence d'un capitalisme de la copie dans les grands pays dits émergents du sud: l'Inde, la Chine, et le Brésil. En 2016, l'industrie pharmaceutique indienne, qui approvisionne en matières premières et en médicaments génériques les marchés mondiaux des nords et des suds, est la $3^{\text {ème }}$ industrie mondiale en volume mais seulement la $14^{\text {ème }}$ en valeur, ce qui pointe la captation de l'essentiel de la valeur par les firmes innovatrices des Etats-Unis et de l'Europe. En 2015, la phyto-chimiste chinoise Tu Yuyou de l'Institut de Médecine Traditionnelle Chinoise à Pékin, reçut le prix Nobel de Médecine, pour la découverte de l'artémisinine, intervenue dans les années 1970. L'artémisinine est le principe actif à la base des nouvelles thérapies de traitement du paludisme recommandées par l'OMS depuis le début des années 2000. En 2016, les analystes financiers remarquèrent l'arrivée de trois sociétés de biotechnologie chinoises parmi les levées de fonds les plus élevées sur les marchés financiers. Il est vrai que la croissance des investissements de $R \& D$ et des brevets en Chine est spectaculaire dans le champ des industries de santé depuis le début des années 2000. En mars 2018, le Ministère de la santé du Brésil autorisait 3 partenariats de développement de produit (PDP) associant des laboratoires publics et privés pour la copie et la fabrication des génériques de la molécule phare de Gilead ${ }^{1}$ pour le traitement de l'Hépatite $C$, le sofosbuvir, et cela malgré la crise politique et économique et l'incertitude des confrontations sur la propriété intellectuelle avec la multinationale. Et pour ce médicament très disputé au nord et au sud, le sofosbuvir, la géographie de sa copie s'est étendue depuis 2015 à l'Egypte, au Maroc, à l'Argentine, à l'Algérie, où la propriété intellectuelle de la molécule était lacunaire, invalidée ou contournée, ainsi qu'à neuf laboratoires indiens qui ont bénéficié de licences volontaires de Gilead, concédées pour faire la part du feu avec les oppositions et pour contrôler les fabricants indiens.

Il faut désormais compter sur les laboratoires des suds pour approvisionner les marchés des grands donateurs globaux pour les médicaments contre le VIH/sida et le paludisme, pour fournir les marchés des génériques en Afrique, en Asie du sud-est, en Amérique latine, mais également dans les pays à hauts revenus, et pour livrer environ $60 \%$ des principes actifs utilisés par

\footnotetext{
${ }^{1}$ Gilead Science est une société de biotechnologie créée en 1987 qui est devenue une des multinationales pharmaceutiques les plus rentables grâce à l'acquisition de médicaments inventés par l'académie ou d'autres sociétés de R\&D. En 2011 elle acheta la Cie Pharmasset, qui avait développé le sofosbuvir, pour 11, 3 Mds de dollars.
} 
l'industrie pharmaceutique américaine et européenne. Le texte revient donc sur : 1) les conditions d'émergence d'un capitalisme de la copie dans les suds à la faveur de la contestation de la propriété intellectuelle des multinationales des nords, cela dès les années 1970 ; 2) les deux modèles de ce capitalisme de la copie, principalement tiré par les besoins domestiques et la politique de santé au Brésil, et principalement tourné vers les exportations sur les marchés mondiaux des matières premières et des génériques pour l'Inde ; 3) les relations entre l'activité de copie et la production de savoirs et d'innovations dans les suds ; 4) les nouvelles confrontations pour le contrôle de la rente globale depuis l'adoption par le Brésil et l'Inde des brevets pharmaceutiques respectivement en 1997 et 2005 ; 5) les circulations et les convergences de luttes au nord et au sud pour l'accessibilité des innovations thérapeutiques, notamment pour les antiviraux contre les hépatites et les anticancéreux, dont l'inflation des prix tend à déstabiliser au nord les couvertures santé déjà fragilisées par la crise de 2008 et au sud à produire de nouvelles restrictions tandis que les projets de couverture universelle promus par l'OMS en 2012 sont encore bien lacunaires.

\section{Réduire l'emprise de la propriété intellectuelle et des multinationales}

Le fait majeur qui a déplacé la géographie des industries de santé est le changement des normes de brevetabilité intervenu quasi-simultanément en Inde et au Brésil, au tout début des années 1970. A l'époque, la critique à l'encontre des multinationales et du contrôle qu'elles exercent sur la technologie mondiale était singulièrement forte, en premier lieu par des économistes des suds, à l'instar de Celso Furtado au Brésil qui soulignait l'urgence de financer des institutions nationales de R\&D pour s'approprier les technologies avancées. En 1986, des experts de la propriété intellectuelle en France notaient la montée des attaques contre le «brevet monopole technologique» à la faveur «de la revendication tiers-mondiste qui veut y voir un instrument de l'impérialisme économique $»^{2}$.

En 1971, le Brésil supprima toute propriété intellectuelle sur les inventions thérapeutiques (il exclut les procédés de fabrication de la brevetabilité alors que les produits étaient déjà non brevetables depuis 1945). Simultanément, le gouvernement restructurait le laboratoire public Farmanguinhos et créait la Centrale des Médicaments (la CEME) pour fournir des médicaments de base à la population pauvre. Dans les années 1980, la CEME subventionna les laboratoires brésiliens publics et privés pour entreprendre la copie des médicaments essentiels, ce qui provoqua des rétorsions commerciales de la part des Etats-Unis de Reagan en 1988.

\footnotetext{
${ }^{2}$ Plasseraud et Savignon, L'Etat et l'invention, La Documentation Française, p 128.
} 
En 1970 la réforme du droit des brevets en Inde supprimait la brevetabilité des produits pharmaceutiques, tout en maintenant des brevets de procédés pour une courte durée de sept années. En plus de la suppression des brevets de produits pharmaceutiques, le gouvernement mit en place des incitations pour se fournir en matières premières domestiques. Cette politique a favorisé l'intégration verticale de l'industrie pharmaceutique indienne, de la synthèse des principes actifs à la formulation des médicaments, ce qui lui permet aujourd'hui de capturer davantage de valeur, alors que l'industrie brésilienne pâtit encore de son manque d'intégration et doit importer la plupart de ses principes actifs d'Inde et de Chine.

Les économistes ont mesuré la croissance vertigineuse de l'industrie des génériques en Inde qui a découlé de l'exclusion des brevets de produits et de la limitation drastique des brevets de procédés entre 1970 et 2005, date à laquelle l'Inde s'est conformée aux accords de l'OMC sur les Aspects des Droits de Propriété Intellectuelle liés au Commerce (ADPIC). En 35 années, la valeur de la production indienne a été multipliée par 150 tandis que le nombre de firmes passait de 1600 à 9000 . Alors que la part de marché des industries nationales avait baissé entre 1952 et 1970 (de 62 à 32\%), en raison de l'emprise grandissante des multinationales qui contrôlaient le flux des innovations (antibiotiques, néo sulfamides, etc.), elle grimpa à $77 \%$ en 2004 , avant la révision de la loi sur les brevets de mars 2005.

Si l'industrie pharmaceutique brésilienne s'était renforcée à la faveur de la seconde guerre mondiale, l'emprise des multinationales s'étendit dès les années 1950 : même en l'absence de brevets de produit, les laboratoires domestiques ne détenaient pas les technologies pour produire les antibiotiques. Les économistes parlent d'un processus de «dé-nationalisation » à la faveur du rachat des laboratoires nationaux par les multinationales ou d'accords de joint-venture. A la fin des années 1960, les firmes à capitaux nationaux ne représentaient plus que $17 \%$ du marché national. En raison de la suppression des brevets de produit et de procédé en 1971 et des politiques de subvention des opérations de « reverse engineering », l'industrie domestique créa de nouvelles capacités technologiques et industrielles dans les années 1980 lesquelles furent remobilisées en réponse à l'épidémie de VIH/sida pour produire des génériques dans les années 1990.

Il faut rappeler un autre cycle d'appropriation des technologies pharmaceutiques par les pays du sud, celui lié à la décolonisation. L'un des pays emblématiques de cette politique est l'Egypte de Nasser qui entreprit à partir de 1956 d'encourager la production locale, d'abord privée, avant de conduire un programme de nationalisation de l'industrie pharmaceutique à partir de 1962. La duplication des technologies pharmaceutiques fut rendue possible par 
l'annulation des brevets portant sur les produits pharmaceutiques par une loi de 1949. En 1975, l'industrie égyptienne produisait $84 \%$ de la consommation nationale. Cette industrie égyptienne, qui a été partiellement privatisée et soumise aux nouvelles lois des brevets de l'OMC au cours des années 1990, constitue aujourd'hui encore une ressource pour produire des médicaments à bon marché contre les épidémies qui affectent le pays, en particulier l'hépatite $\mathrm{C}$ qui touche au moins $15 \%$ de la population. En 2015, les laboratoires égyptiens ont été en mesure de produire le nouvel antiviral contre l'hépatite $\mathrm{C}$ du laboratoire Gilead, le sofosbuvir, pour un prix de $\$ 300$ dollars alors que le laboratoire propriétaire l'offrait au Ministère de la Santé pour $\$ 900$ dollars. Entre temps, l'office des brevets égyptien, pressé par les associations de patients, avait refusé le brevet de Gilead.

On trouve encore l'héritage de ces politiques dans plusieurs pays africains, à l'instar du Maroc, du Ghana de Nkrumah, de la Tanzanie de Nyerere, qui engagèrent des politiques de soutien aux industries pharmaceutiques dès le début des années 1960, en créant des laboratoires publics ou en adoptant des lois favorables à la formation d'un secteur privé occupé par des laboratoires nationaux ou des établissements de firmes multinationales. Si les médicaments indiens occupent $40 \%$ des enregistrements en Tanzanie, les producteurs locaux en représentent un peu moins de $20 \%$ et capturent 30\% de parts de marché. Le Maroc héberge une industrie pharmaceutique en croissance qui entend fournir les marchés de l'Afrique subsaharienne.

La politique des médicaments essentiels de l'OMS lancée par Halfdan Malher $^{3}$ ans les années 1970 intégrait le développement de la production locale dans les pays en développement, via la création d'usines de formulation. Toutefois, la fédération internationale de l'industrie pharmaceutique ne souhaitait pas que la production locale soit une priorité. Elle privilégiait l'amélioration des systèmes locaux de distribution et l'approvisionnement du secteur public en médicament et consentit à des expériences limitées de distribution de médicaments dans quelques pays d'Afrique et d'Asie du sud-est.

Ainsi, ce sont davantage les politiques de «nationalisation » des industries de santé à l'initiative de plusieurs Etats, en premier lieu l'Inde, le Brésil, la Chine, qui visaient l'appropriation locale des technologies extérieures par des firmes pharmaceutiques publiques ou privées bénéficiant de politiques de propriété

\footnotetext{
${ }^{3}$ Halfdan Malher fut le Directeur Général de l'OMS de 1973 à 1988. Il inspira une nouvelle vision de la santé publique fondée sur la Santé Primaire (Déclaration d'Alma Ata en 1978), au lieu de se focaliser sur des programmes verticaux d'éradication de telle ou telle maladie, et sur la rationalisation du marché des médicaments (Listes des Médicaments Essentiels publiés depuis 1977 jusqu'à nos jours).
} 
intellectuelle ouvrant l'espace de la copie, qui expliquent les changements de la géographie industrielle depuis les années 1960 et 1970.

\section{Le capitalisme de la copie entre besoins domestiques et marchés mondiaux}

Le Brésil et l'Inde permettent de décrire deux modèles divergents de développement de ces industries de santé au Sud.

L'intégration entre les besoins de santé et la politique industrielle fut particulièrement forte lorsque des grandes villes du Brésil puis le gouvernement fédéral entreprirent, sous la pression des associations de patients « $\mathrm{VIH} /$ sida » et de leurs avocats, de distribuer gratuitement des antiviraux, au début des années 1990. La tension sur les prix alors très élevés de l'AZT puis des trithérapies à acquérir pour les programmes de distribution universelle incita très vite les industriels brésiliens à réaliser le reverse engineering de ces médicaments ${ }^{4}$. C'est d'ailleurs un laboratoire privé, essaimé de l'université fédérale de Rio, Microbiologica, qui mit le premier l'AZT sur le marché, avant qu'un laboratoire public du Nordeste, le Lafepe, ne le suive. C'est ensuite le laboratoire fédéral Farmanguinhos qui étendit cette politique de copie, en coopération étroite avec des laboratoires privés, qui fournissaient les principes actifs aux laboratoires publics chargé de produire les formulations, tandis que des universitaires épaulaient les uns et les autres.

Cette politique a conduit à une expansion des firmes privées et publiques de médicaments génériques, au point que certaines d'entre elles furent rachetées au début des années 2010 par des multinationales comme Aventis. Il en est résulté une multiplication des partenariats technologiques gouvernés par l'Etat, qui associent des laboratoires publics et des laboratoires privés, nationaux et parfois multinationaux, pour approvisionner le marché domestique. Cette production pilotée par le Ministère de la santé est pour l'essentiel destinée aux besoins du pays : aucun laboratoire brésilien n'a candidaté jusqu'ici pour obtenir une pré-qualification de l'OMS, étape indispensable pour accéder au marché des grands donateurs globaux, en premier lieu du Fonds Mondial contre le Sida, la tuberculose et la malaria; situation inverse de celle de l'industrie indienne qui a capturé les marchés mondiaux de génériques.

L'économiste Sudip Chaudhuri a parfaitement analysé le rôle de l'industrie indienne comme "pharmacie du monde ${ }^{5}$. Ce rôle est apparu dans l'espace public mondial lorsque la firme Cipla a proposé en 2001 une trithérapie

\footnotetext{
${ }^{4}$ Le reverse engineering (ingénierie inverse en français) consiste à reconstituer le savoir et le savoirfaire inscrits dans un objet fabriqué, ici un médicament, pour le reproduire.

5 The WTO and India's Pharmaceuticals Industry (Patent Protection, TRIPS, and Developing Countries), Oxford University Press, 2005.
} 
à dose fixe de son invention pour 1 dollar par jour, alors que le prix des trithérapies propriétaires, sans dose fixe, s'élevait alors à 10000 dollars par an. Cipla a développé alors un véritable activisme thérapeutique pour promouvoir l'accès au médicament dans les suds. Les principaux laboratoires indiens qui se sont lancés dans la production des antirétroviraux ont très vite entrepris d'obtenir des certifications internationales auprès de la FDA ou du nouveau système de préqualification mis en place par l'OMS pour garantir la qualité des copies destinées à traiter le sida, la malaria et la tuberculose. Le Directeur Général de Cipla exposait ainsi l'enjeu de la pré-qualification OMS : cela « prouve que nous [Cipla] adhérons aux bonnes pratiques de fabrications sur un pied d'égalité avec d'autres compagnies. [...] Maintenant les multinationales ne pourront plus nous apostropher de cette manière : Ils sont Indiens, ils viennent du Tiers-Monde, la qualité doit être douteuse ». En 2010 les firmes indiennes représentaient $68 \%$ des 267 médicaments pré-qualifiés ${ }^{6}$, alors que les firmes brésiliennes, tournées vers leur marché domestique, ne détenaient aucune préqualification. De ce fait, les firmes indiennes peuvent vendre sur le marché des donateurs globaux, comme le Fonds Mondial, qui exigent la pré-qualification. En 2008, les fabricants indiens fournissaient $90 \%$ des antirétroviraux utilisés dans le traitement des infections à VIH dans les pays du sud.

Outre leur large captation du marché des donateurs globaux, avec des médicaments certifiés avec des standards de qualité très élevés, les firmes indiennes sont largement présentes sur les marchés locaux africains ou d'Asie du sud-est, avec des standards éventuellement moins élevés. Sudip Chaudhuri, qui a conduit des enquêtes au Ghana et en Tanzanie, a montré la forte pénétration des génériques indiens sur les marchés africains, soit 40\% des médicaments enregistrés en Tanzanie en 2010. Il faut ajouter l'importance des circulations de techniciens indiens dans les usines africaines ainsi que l'achat de machines indiennes dont le prix est sensiblement plus abordable que les machines américaines ou italiennes.

Si les compétences des laboratoires indiens sont parfois controversées par les laboratoires du nord, ces derniers recourent en fait massivement aux firmes de chimie pharmaceutique indiennes pour s'approvisionner en substances actives en économisant sur les coûts de fabrication. L'industrie indienne s'est spécialisée dans l'exportation de matières premières pharmaceutiques vers les Etats-Unis et l'Europe qui constatent depuis quelques années une dépendance parfois problématique: "Plus des trois quarts de la production de principes actifs et presque un quart de la production des formulations pharmaceutiques

\footnotetext{
${ }^{6}$ La «préqualification» OMS : origines, déploiement et impacts sur la disponibilité des antirétroviraux dans les pays du Sud, C. Lantenois et B. Coriat, Sciences Sociales et santé, 1, vol 14, 2014.
} 
sont exportés. Les Etats-Unis, qui ont les exigences réglementaires les plus strictes, sont devenus la première destination exportatrice de l'Inde » (Chaudhuri, 2005). Récemment, le Ministère indien de la santé s'est inquiété de la dépendance des producteurs locaux vis à vis des importations chinoises de principes actifs qui pratiquent un dumping très agressif. Face aux risques de dépendance et de ruptures d'approvisionnement de principes actifs indiens ou chinois, le Ministère de l'Industrie français entrepris de relocaliser certaines synthèses de principes actifs pharmaceutiques.

Tandis que l'industrie des génériques du Brésil, qui a connu une forte croissance dans les années 2000, est tirée par les achats du Système Unique de Santé et par la croissance des marchés privés de médicaments similaires à ceux de marque, l'industrie indienne des génériques est tirée principalement par des exportations vers les nords et les suds. Un des paradoxes les plus notables réside en Inde dans le découplage entre la production nationale de génériques et les besoins de santé des populations pauvres : «L'Inde exporte des médicaments d'importance vitale vers les pays en développement et fournit des médicaments de qualité aux pays riches et à des prix abordables. En dépit de cette performance apparemment louable, des millions de ménages indiens n'ont pas accès aux médicaments » (Rapport du groupe d'experts de haut niveau sur la couverture maladie universelle pour l'Inde, 2011). Quant au Brésil, l'insuffisance des dépenses publiques de santé, accentuée par la crise ouverte en 2015, fragilise l'accès de nombreux brésiliens aux médicaments.

\section{Entre copies et innovations}

La copie de molécules sophistiquées, à l'instar des antiviraux contre le VIH ou le VHC, a produit de nouveaux savoirs au sein des firmes imitatrices qui ont dû renforcer leurs activités de $\mathrm{R} \& \mathrm{D}$, internes et en partenariat avec les universités. Les chimistes indiens ont acquis une forte expertise, ce qui leur a permis de réduire les temps de « reverse engineering » et de raccourcir les délais de diffusion des copies après la mise sur le marché des nouveaux médicaments aux Etats-Unis : «Des études récentes portant sur 17 molécules mises sur le marché depuis 1994 tendent à montrer que l'industrie indienne est devenue experte du reverse engineering des nouveaux produits, si bien que les patients bénéficient des nouveaux produits avec des délais de plus en plus brefs depuis la date d'introduction aux États-Unis » ${ }^{7}$.

\footnotetext{
${ }^{7}$ Scherer \& Watal : «Post-Trips Options for Access to Medicines in Developing countries”, 2001, 97 pages.
} 
De cinq ans en moyenne avant 1985, le temps de la copie est passé à deux ans dans les années 1990. En 2005 Roche mit en cause la capacité de Cipla pour dupliquer le Tamiflu contre la grippe : Cipla reconnut la difficulté de la tâche et mit en avant l'expertise acquise dans la reproduction des ARVs: "Ce n'est pas une synthèse facile, mais notre société a l'habitude de fabriquer des choses difficiles ... L'une des étapes de la zidovudine, par exemple, utilise de l'azide de sodium, et c'est ce que Roche a qualifié de dangereux et de dangereux. Nous le faisons au moins depuis 14 ans. Tout cela, c'est de la chimie " (Yusuf K Hamied, Forum Mondial pour la Recherche en Santé, 21 october 2005). Et à l'automne 2005, Cipla mettait sur le marché un générique du Tamiflu.

La copie ne se réduit jamais à la simple duplication d'une recette codifiée dans un brevet ou une publication. Comme toute reproduction de technologie, elle suppose de surmonter l'incomplétude des descriptions, a fortiori celles d'un brevet écrit avec le souci d'étendre les revendications et de réduire les informations divulguées. L'imitateur doit donc procéder pas à pas, en alternant lecture des brevets et manipulations en laboratoire, pour reconstituer les procédés de fabrication. De plus, la compétitivité du producteur de génériques réside dans les améliorations qu'il introduit pour augmenter la productivité de la synthèse. Si bien que les producteurs brésiliens ou indiens inventent sans cesse de nouvelles routes de synthèse, tenues secrètes ou brevetées.

Une des spécialités des producteurs indiens et brésiliens a résidé dans la mise au point de nouvelles combinaisons à dose fixe : ils ont utilisé au début des années 2000 la possibilité de librement associer des molécules libres de droit, association difficiles et coûteuses à réaliser dans une situation où les molécules brevetées sont réparties entre plusieurs propriétaires. Le coût de maître dans cette lignée d'invention fut la formulation de la Triomune par le laboratoire Cipla, la première combinaison à dose fixe contre le VIH/sida, mise sur le marché en 2001. Elle fut plébiscitée par les patients qui voyaient leurs traitements facilités et son prix modique tira les prix mondiaux des trithérapies du sida vers le bas.

Des firmes brésiliennes ont accumulé un portefeuille de brevets depuis le début des années 2000 sur de nouvelles formulations, sur des molécules dérivées ou hybrides, ou carrément sur de nouvelles familles de molécules, dans le champ du VIH, de la malaria ou encore de la tuberculose. Une des firmes les plus remarquables du Brésil, Microbiologica, qui introduisit l'AZT dans le pays, participa au développement des médicaments contre les hépatites virales aux côtés des chercheurs américains ou français qui les mirent au point au début des 
années $2000^{8}$. Elle détient par exemple un brevet de procédé cité dans le brevet de Gilead sur le sofosbuvir.

J'ai évoqué en introduction la découverte de l'artémisinine par les chercheurs chinois au début des années 1970. Cette invention procède d'un autre mode de production qui repose sur la combinaison systématique de la pharmacopée traditionnelle et de la chimie. L'artémisinine résulte aussi d'un vaste processus d'invention collective qui mobilisa des centaines de chercheurs académiques et industriels dont les premiers produits ne furent pas brevetés, ce qui facilita leur reproduction et leur libre combinaison pour former des combinaisons à base d'artémisinine. Cependant, la première combinaison à dose fixe développée par les chimistes chinois, et encore aujourd'hui la plus utilisée, artéméther et luméfantrine, fut couverte par un brevet chinois en 1990 et par un brevet international en 1991, en co-propriété par l'Académie Militaire des Sciences Médicales de Chine et par Ciba Geigy. Ce fut la multinationale qui commercialisa ce médicament sur les marchés mondiaux à partir du début des années 2000 et qui s'en appropria l'essentiel de la valeur, nonobstant une partie de la fabrication réalisée dans une usine en Chine.

Les capitalismes de la copie d'Inde, de Chine ou du Brésil ont au fil du temps renforcé leurs capacités d'innovation, même si leur part dans le flux des inventions demeure limité ${ }^{9}$. Les grands laboratoires de génériques ont augmenté leurs dépenses de $R \& D$. Une dizaine de grands laboratoires indiens se sont engagés au début des années 2000 dans l'invention de nouveaux médicaments, souvent des modifications de molécules existantes, et ont déposé de plus en plus de brevets, y compris aux Etats-Unis. Toutefois, ils en sont encore réduits à passer des accords avec les multinationales des nords pour mettre leurs inventions sur le marché international. La croissance la plus remarquable est celle des investissements de R\&D et des dépôts de brevets par la Chine : les flux de nouvelles molécules en essais cliniques et mises sur le marché augmentent régulièrement depuis 2000, même si plus de 9 firmes sur 10 se consacrent aux génériques. L'extension de la couverture santé engagée en Chine en 2009 pourrait être un puissant support de l'innovation comme elle l'a été dans les pays industrialisés du nord après 1945.

\footnotetext{
${ }^{8}$ Health Innovation and Social Justice in Brazil, Cassier Maurice, Correa Marilena, Eds, 2018, Palgrave Macmillan.

${ }^{9}$ From imitation to innovation : a Study of Chinese Drugs and Relevant National Policies, Jingxi Ding, Yajiong Xue' Huigang Liang' Rong Shao',Yongfa Chen, Journal of Technological Management and Innovation, 2011, v 6, issue 2.
} 


\section{Les nouvelles confrontations pour le contrôle de la rente globale}

Tandis que les capitalismes de la copie renforçaient leur base dans les grands pays émergents, les multinationales et les pays innovateurs n'ont eu de cesse de fermer l'espace de l'imitation. Ce fut justement le grand dessein des accords sur la propriété intellectuelle liée au Commerce de l'OMC entrés en vigueur en 1995, puis des accords bilatéraux de libre-échange négociés jusqu'à aujourd'hui. Si l'Inde a pleinement utilisé la période de transition de dix ans autorisée par les ADPIC pour se conformer aux brevets de produit pharmaceutique, ce qui lui a permis d'accumuler des savoirs et de renforcer ses industries, le Brésil, pressé par les Etats-Unis, a fermé son espace de copie dès 1997, sauf à défaire des brevets par des licences obligatoires ou des oppositions à l'initiative du Ministère de la santé, des producteurs de génériques et des associations de patients. Si bien que depuis 2005, date de l'entrée en vigueur des brevets de médicaments en Inde et dans de nombreux pays en développement, sauf les pays africains à bas revenus où la copie reste licite, ce qui est un atout aujourd'hui, les multinationales ont les outils juridiques pour contrôler une rente pharmaceutique de plus en plus globalisée.

L'exemple le plus emblématique de l'existence de cette rente globale est le contrôle du marché très sophistiqué mis en place par la société étatsunienne Gilead Science sur les différents antiviraux contre l'hépatite $\mathrm{C}$ qu'elle a mise sur le marché depuis 2014. Après avoir fixé les prix du sofosbuvir les plus élevés possibles selon l'évaluation des capacités des payeurs de l'Amérique du Nord et de l'Europe, jusqu'à inquiéter la viabilité de la sécurité sociale en France qui devait s'acquitter jusqu'ici de 41000 euros pour un traitement, Gilead a étendu une barrière de brevets très dense dans tous les pays producteurs, non sans quelques trous comme au Maroc où une firme a pu librement s'engager dans la copie. En septembre 2014, elle a concédé des licences volontaires à des firmes indiennes pour produire des génériques pour une liste fermée de pays à bas revenus qui exclut des pays dits intermédiaires comme le Brésil et la Thaïlande. En deux années, 2014 et 2015, Gilead a engrangé 32 Mds de dollars avec deux médicaments contre l'hépatite $\mathrm{C}$, le sofosbuvir et une combinaison à base de sofosbuvir, Harvoni. Alors que ses revenus doublaient, sa profitabilité était multipliée par cinq et la hiérarchie des grands laboratoires fut bouleversée.

Les enquêtes récentes de $\mathrm{S}$. Chaudhuri conduites en Inde ${ }^{10}$ montrent le renforcement des positions des multinationales du nord depuis le retour des brevets de médicaments. Les multinationales rachètent des firmes indiennes, augmentent leurs importations de formulations à prix élevés produites en Europe

\footnotetext{
${ }^{10}$ Impacts of Product Patents on Pharmaceutical Market Structure and Prices in India, sept 2018, 50 p.
} 
et introduisent des médicaments innovants à des prix prohibitifs pour une large partie de la population, particulièrement dans le champ du cancer.

Face à cette offensive des multinationales, les fabricants de génériques et les associations de patients ont multiplié les oppositions et ont réussi à faire invalider de nombreux brevets (43 sur un échantillon de 135 nouvelles molécules). En avril 2013, la Cour Suprême Indienne a pris un arrêt très remarquable $^{11}$ qui a confirmé l'invalidation du brevet de Novartis sur un anticancéreux très onéreux, le Glivec, et qui a défendu le droit d'un Etat d'interpréter le droit des brevets en ayant le souci de défendre la santé publique. Le Brésil de Lula, fort de sa politique d'accès universel et de la croissance de son industrie des génériques, avait en son temps, en mai 2007, décidé d'une licence obligatoire pour nationaliser un antiviral de Merck, l'efavirenz.

\section{Circulations mondiales et convergences des luttes sur les prix et l'accès aux traitements}

Si la rente pharmaceutique s'est globalisée depuis le milieu des années 1990, les luttes pour l'accès aux traitements se sont mondialisées de manière symétrique, notamment depuis le procès de Prétoria en Afrique du sud initié en 1998. Tandis que la Campagne d'Accès aux Médicaments Essentiels fondée par Médecins Sans Frontières en 1999 implantait des délégués dans des pays jugés stratégiques pour résister aux brevets de médicaments et pour décider de licences obligatoires, en premier lieu le Brésil et l'Afrique du sud, les associations de patients des pays du sud ont très notablement renforcé leur contre-expertise dans le champ de la propriété intellectuelle. Ainsi en novembre 2008 se tenait un séminaire à Rio de Janeiro, avec le soutien du laboratoire fédéral Farmanguinhos, qui réunissait des associations de patients et des juristes «alternatifs » d'Inde et du Brésil, pour enseigner la méthode de rédaction des oppositions aux brevets. En 2006, une association de patients du VIH/sida s'était jointe à une association indienne pour déposer une opposition qui a fait tomber un brevet de Gilead sur un antiviral très utilisé le Tenofovir.

L'arrivée de la nouvelle classe des antiviraux contre l'hépatite C en 2014 a remobilisé ces réseaux de collaboration pour contester les brevets ou pour demander leur suspension temporaire par des licences obligatoires. Les oppositions se sont alors mondialisées et coordonnées, au Brésil, en Inde, en Egypte, en Europe, aux Etats-Unis, pour échanger des arguments pour faire opposition aux brevets sur le sofosbuvir. Médecins du Monde, qui est intervenue pour la première fois dans le champ de la propriété intellectuelle, a déposé une opposition à l'Office Européen des Brevets à Munich, bientôt rejointe par MSF.

\footnotetext{
${ }^{11}$ Novartis vs Union of India, Supreme Court of India, april 01, 2013.
} 
MDM et MSF se sont informés des oppositions déposées au Brésil et en Inde sur la même molécule. La fondation DNDi, créée en 2003 par MSF et plusieurs institutions publiques de recherche au Brésil, en Inde, au Kenya et en Malaisie, a développé un programme pour favoriser l'accès aux nouveaux antiviraux contre l'hépatite $\mathrm{C}$ : elle s'est notamment rapprochée de deux fabricants en Egypte et en Argentine pour convenir de la production de génériques a des prix très bas (moins de 300 dollars le traitement).

Les revendications pour l'accès et la maitrise des prix des innovations thérapeutiques circulent entre le nouveau collectif inter-associatif créé en France en juin $2018^{12}$, la Campagne d'Accès aux Médicaments Essentiels de MSF, les associations du Brésil, d'Inde, de Thaillande assistée par des experts en droit et en pharmacie. Plusieurs pays européens, récemment le Parlement norvégien, débattent de l'utilisation de la licence obligatoire pour endiguer les prix exorbitants des nouveaux traitements contre les hépatites, les cancers et les maladies orphelines. Les controverses et les luttes pour les licences obligatoires, focalisées au début des années 2000 sur les suds, ont aujourd'hui gagné les nords.

12 «Médicament et progrès thérapeutique : garantir l'accès, maîtriser les prix, la Contribution de la Société Civile au débat public en France » MSF, Prescrire, MDM, Aides, Que Choisir, La ligue contre le Cancer, France Assos Santé, UAEM. 